\title{
Analysis of Human Motion Variation Patterns Using UMPCA
}

Hadi Ibrahim Masoud

Yaser Zerehsaz (Corresponding author)

Jionghua (Judy) Jin 


\section{Analysis of Human Motion Variation Patterns Using UMPCA}

The rapid development of motion capture technologies has greatly increased the use of human motion data in many applications. This has increased the demand to have an effective means to systematically analyze those massive data in order to understand human motion variation patterns. This paper studies one typical type of motion data, which are recorded as multi-stream trajectories of human joints. Such a high dimensional multi-stream data structure makes it difficult to directly perform visual comparisons or simply apply conventional methods such as PCA to capture the variation of human motion patterns. In this paper, a high order array (tensor) is suggested for data representation, based on which the Uncorrelated Multilinear Principal Component Analysis (UMPCA) is applied to analyze the variation of human motion patterns. A simulation study is presented to show the superiority of UMPCA over PCA in preserving the crosscorrelation among multi-stream trajectories. The effectiveness of UMPCA is also demonstrated using a case study for analyzing vehicle ingress test data.

Keywords: human motion; variation analysis; UMPCA

\section{Introduction}

In recent years, increasing interest in the analysis of human motion data has been driven by a wide range of applications in different fields. In the athletics field, for example, Li et al. (2006) presented a multistep algorithm to automatically detect and recognize athletes' sporting actions in a long video with a dynamic background. Knudson (2013) introduced the concept of Qualitative Movement Diagnosis (QMD) to improve athletes' performance and reduce their risk of injury by visually observing video recordings of their motion. In the medical field, gait analysis, which uses motion data to analyze patients' walking patterns, is used to assist doctors' clinical diagnosis and treatment decisions. For example, Kay et al. (2000) discussed the impact of postoperative gait analysis on the assessment of treatment outcomes and planning on- 
going care. In the automotive industry, Masoud et al. (2016) presented a systematic framework for using human ingress motion trajectories to predict customers' ingress discomfort ratings for improving vehicle design. Although these applications may have different objectives in terms of the usage of motion data, some common questions are often encountered during data analysis. For example, what are the typical motion variation patterns among different participants? Which time segments in a long video would be related to motion patterns of interest? Which particular joints and moving directions will reflect a normal or abnormal motion pattern of interest? The main goal of this paper is to use a systematic way to provide reasonable answers for these sorts of questions. In other words, analyzing human motion variation patterns in the ingress motion means to find the joints, motion directions $(\mathrm{X}, \mathrm{Y}$ and $\mathrm{Z})$ and time segments that suggest the highest variability among all subjects performing the ingress motion. A noteworthy point is that variability in this context is defined among different subjects performing the ingress motion. Furthermore, it is important to capture the crosscorrelation structure among the joints in order to have more thorough understanding of the motion's mechanism, which is studied in detail in Section 3.

There are various ways to analyze human motion variation patterns. One simple way to analyze motion patterns of tested subjects is to replay the recorded motion videos multiple times and use visual comparison. In Cook et al. (2003), for instance, gait analysis experts visually compared 3D joint motion trajectories of patients having cerebral palsy to the "normal" motion trajectories to assess patients" need for surgery. This approach is effective in the existence of simple motions where the observer knows what type of pattern they are looking for (i.e. the walking motion of an unhealthy individual compared to that of a healthy one). However, in many other applications, such as vehicle ingress, the motion data is much more complex. The ingress motion data 
involves multiple joints moving in a 3D space. As shown in Figure 1(a), the ingress motion data of one subject are recorded for numerous joints such as ankles, elbows, back vertebrae etc., where the location of each joint over time in 3D moving directions is referred to as a joint trajectory, as shown in Figure 1(b). In this paper, the trajectories of these multiple joints are referred to as multi-stream trajectory data. Considering such a high dimensional data, it is extremely cumbersome, if even possible, to extract the motion patterns among different subjects by visually comparing the recorded motion videos of individual subjects. Consequently, the conclusions regarding motion patterns via visual comparison could be highly subjective. Therefore, having an effective methodology to automatically analyze motion variation patterns is critically needed for reducing tedious data exploration efforts and avoiding subjective bias.

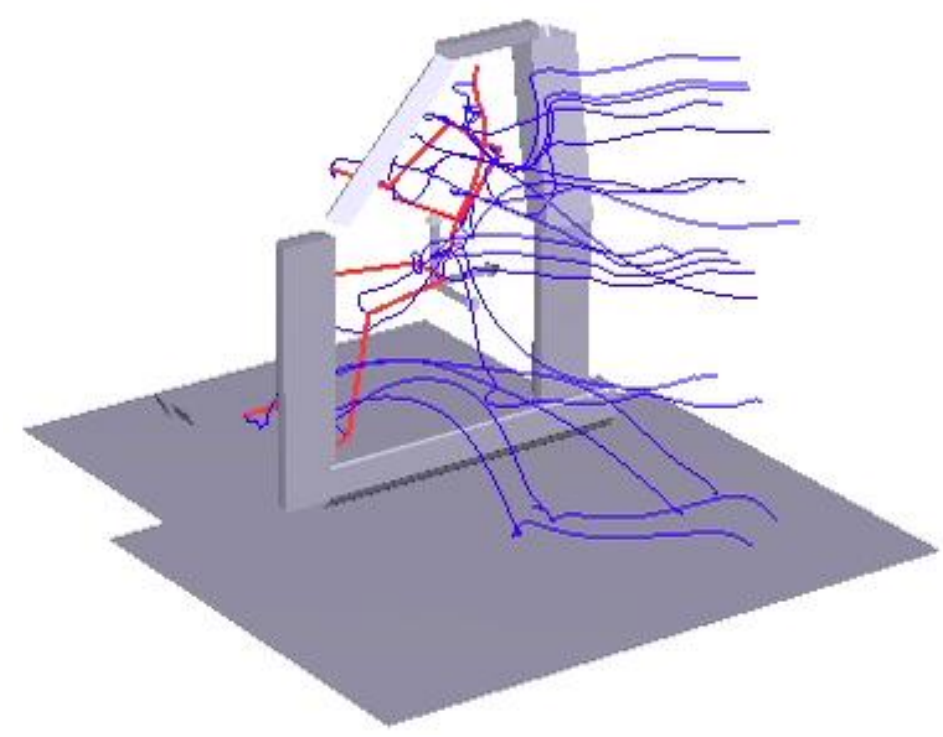

(a) Multi-stream trajectories 


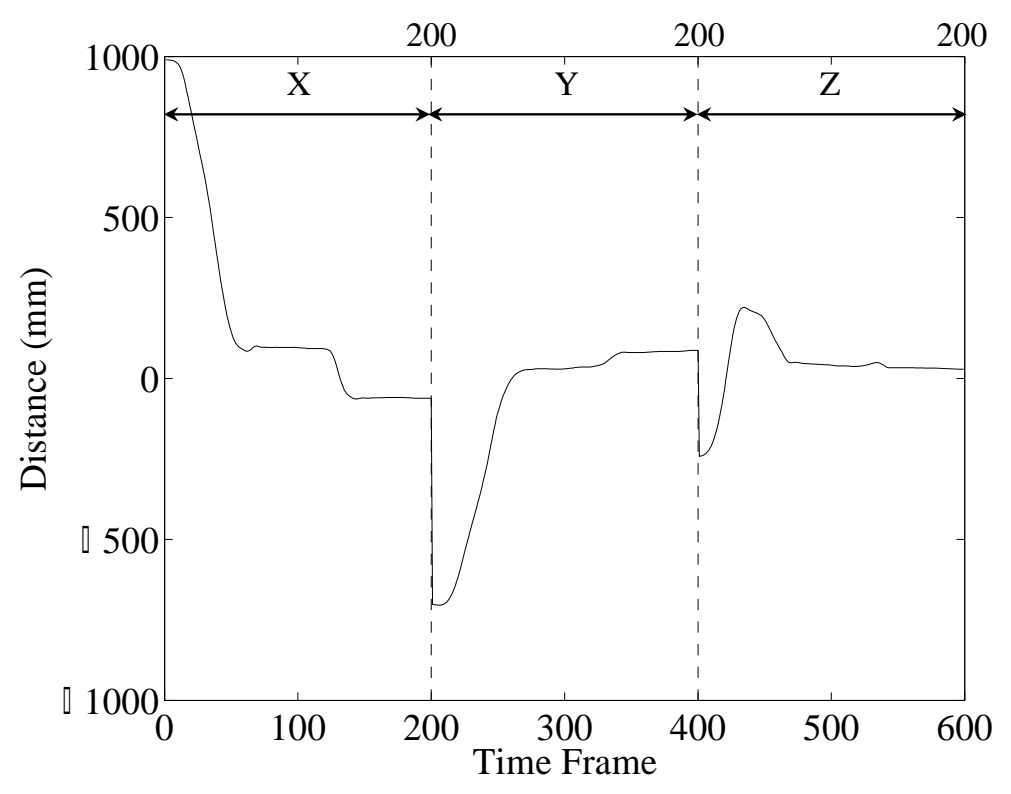

(b) The right ankle joint trajectory

Figure 1: Ingress motion data of one participant

Principal Component Analysis (PCA) is a commonly used method for analyzing variation patterns. If the analysis of motion data is based on a single trajectory, PCA can be applied directly. For example, the motion data of the right ankle joint trajectory shown in Figure 1(b) can be represented by a matrix, where each row vector represents the right ankle joint trajectory of one participant, and the number of rows represents the number of tested participants. Without manipulating the structure of the data, it is not plausible, however, to directly apply the conventional PCA method to multistream trajectories. One naïve way to apply PCA to such data is to combine the multistream trajectories into one high-dimensional vector before applying PCA. For example, if the ingress dataset has $\mathrm{N}$ participants with $\mathrm{K}$ joints recorded for each participant, and each joint trajectory has $\mathrm{M}$ data points, then the new dataset will be represented by a matrix of size $\mathrm{N} \times \mathrm{MK}$. This means that we combine the M-dimensional vectors of all the $\mathrm{K}$ joints for one subject together and consider them as one vector. PCA can subsequently be applied to this matrix to estimate the eigenvectors and extract the significant 
variation patterns indicated by larger eigenvalues. A PCA analysis of this kind is known as stack-up PCA (Mason et al. 2001, Bharati et al. 2004). Using this stack-up method on human motion data arises two issues that affect the performance of PCA method in providing accurate estimates of the eigenvectors; thus misrepresenting the true variation patterns. First, the number of variables (columns) will highly likely exceeds the number of subjects (rows) i.e. MK > N. Second, it is obvious that some of the joints are highly correlated. In the ingress motion context, this means that the locations of joint $\mathrm{A}$ in $\mathrm{X}, \mathrm{Y}$ and $\mathrm{Z}$ directions (trajectories) are correlated to those of joint $\mathrm{B}$, for instance. In this case, if joint A's motion has high variability across the subjects, then joint B must have the same behaviour. This will help find groups of joints that are highly variable in a specific part of the motion; thus having more interpretable results. The problem of stack-up PCA is that since all the joints are combined together, it fails to capture the correlation specially if there is a negative correlation among some joints while the other joints are positively correlated. This problem will be thoroughly discussed and demonstrated in Section 3.

Several authors have pointed out the inefficacy of the stack-up PCA method in these situations (He et al. 2005, Paynabar et al. 2013, Yan et al. 2015). These issues motivate us for utilizing a novel methodology that can analyze the variation patterns without manipulating the correlation structure of the data. Therefore, another type of data representation avoiding the stack-up procedure can be more effective for variation analysis.

In this paper, a high-order array is used to represent the multistream trajectory data, and the Uncorrelated Multilinear Principal Component Analysis (UMPCA) method (Lu et al. 2009) is subsequently applied to analyze variation patterns in human motion. It is then shown that using a high-order representation followed by the UMPCA 
method can preserve the original spatiotemporal correlation structure of the multistream structured data; therefore, it can provide more efficient dimension reduction and feature extraction compared to the PCA method.

The remainder of this paper is organized as follows. Section 2 provides a brief review of the UMPCA method and considers how this method can be used for human motion analysis. In first part of Section 3 a simulation study is presented to demonstrate the superiority of the UMPCA method over PCA in capturing the variation of human motion patterns in multistream datasets. In the second part of Section 3, the use of UMPCA is illustrated for selecting a few important joints, directions, and time segments from massive ingress motion data, which contribute most significantly to motion variations among the tested participants. Section 4 provides concluding remarks.

\section{Methods}

\subsection{Basic Notation of Multistream Algebra}

This section introduces the basis of using a high-order tensor representation for multistream trajectory data. A tensor is a multidimensional array that can be used to represent data with more than two dimensions. Each dimension of a tensor is called a mode or order. A tensor is denoted by $\mathcal{X}^{\mathrm{I}_{1} \times \mathrm{I}_{2} \times \ldots \mathrm{I}_{\mathrm{R}}}$, where $\mathrm{I}_{\mathrm{r}}(\mathrm{r}=1, \ldots, \mathrm{R})$ indicates the number of elements in the $\mathrm{r}^{\text {th }}$ mode. For example, human motion data can be represented by a third-order tensor denoted by $\mathcal{X}^{\mathrm{K} \times \mathrm{M} \times \mathrm{N}}$, where $\mathrm{K}, \mathrm{M}$, and $\mathrm{N}$ are the number of elements in the joint, trajectory, and participant modes, respectively. Although there are different ways of projecting a tensor to a vector, this research focuses on the tensor-to-vector (TVP) method introduced by Lu et al. (2009). Briefly, TVPs are multiple projections of a tensor-to-scalar yielding a vector. In each projection, the TVP method projects the matrix of K-joints' trajectories for each participant to a 
scalar using Elementary Multilinear Projections (EMPs). An EMP is used in a series of mode-r tensor-to-vector products with $\mathrm{r}=1,2$. In other words, the second-order tensor representing each participant's joints trajectories can be projected to a scalar $\mathrm{z}$ as $\mathrm{z}=\mathcal{X} \times_{1} \mathrm{v}_{\mathrm{p}}^{(1)^{\mathrm{T}}} \times_{2} \mathrm{v}_{\mathrm{p}}^{(2)^{\mathrm{T}}}$, where $\mathrm{v}_{\mathrm{p}}^{(1)} \in \mathbb{R}^{\mathrm{K}}$ and $\mathrm{v}_{\mathrm{p}}^{(2)} \in \mathbb{R}^{\mathrm{M}}$ are the $p^{\text {th }}$ projection vectors corresponding to the joint and trajectory modes, respectively. If P EMPs are considered for projection, these EMPs can be sequentially used for tensor-to-scalar projections. As $\mathrm{N}$ participants are performing the ingress motion, using $\mathrm{P}$ EMPs leads to $\mathrm{N}$ projected (transformed) vectors:

$$
\mathrm{z}^{\mathrm{n}} \in \mathbb{R}^{\mathrm{P}}=\left\{X \times_{1} \mathrm{v}_{\mathrm{p}}^{(1)^{\mathrm{T}}} \times_{2} \mathrm{v}_{\mathrm{p}}^{(2)^{\mathrm{T}}}\right\}_{\mathrm{p}=1,2, \ldots \mathrm{P}} \text { with } \mathrm{n}=1,2, \ldots, \mathrm{N}
$$

\subsection{Uncorrelated Multilinear Principal Component Analysis (UMPCA)}

Tensor decomposition has been widely studied in literature, and many techniques to factorize a tensor have been introduced. A detailed review on various decomposition techniques is presented in Kolda and Bader (2009).

This paper aims to apply the UMPCA method proposed by Lu et al. (2009) to decompose the ingress data with the purpose of detecting the most critical segments of the motion. This section briefly explains the essence of the UMPCA method. UMPCA takes advantage of the TVP method to project each tensor to a vector. Analogous to PCA, the objective of UMPCA is to obtain the EMPs (projection vectors) while maximizing the variance of projected vectors. As explained in Figure 2, by exploiting $\mathrm{P}$ EMPs, UMPCA takes the ingress motion tensor $\mathcal{X}^{\mathrm{K} \times \mathrm{M} \times \mathrm{N}}$ and projects it to a lowdimensional subspace in $\mathrm{R}^{\mathrm{p}}$, which is similar to the principal components' subspace in PCA (PC scores) with $\mathrm{P}<\min [\mathrm{K}, \mathrm{M}, \mathrm{N}]$ (Lu et al. 2009). It is noteworthy to mention that similar to PCA, the first projection vector in each mode $v_{1}^{(r)} ; r=1,2$, is the direction of the highest variability of the data in mode $r$. To obtain the direction for the 
second highest variation in the data, the next projection vector $v_{2}^{(r)}$ must be perpendicular to the first one in order to give the most possible amount of information about the variation of data in mode $r$. This is exactly the case in the PCA method.

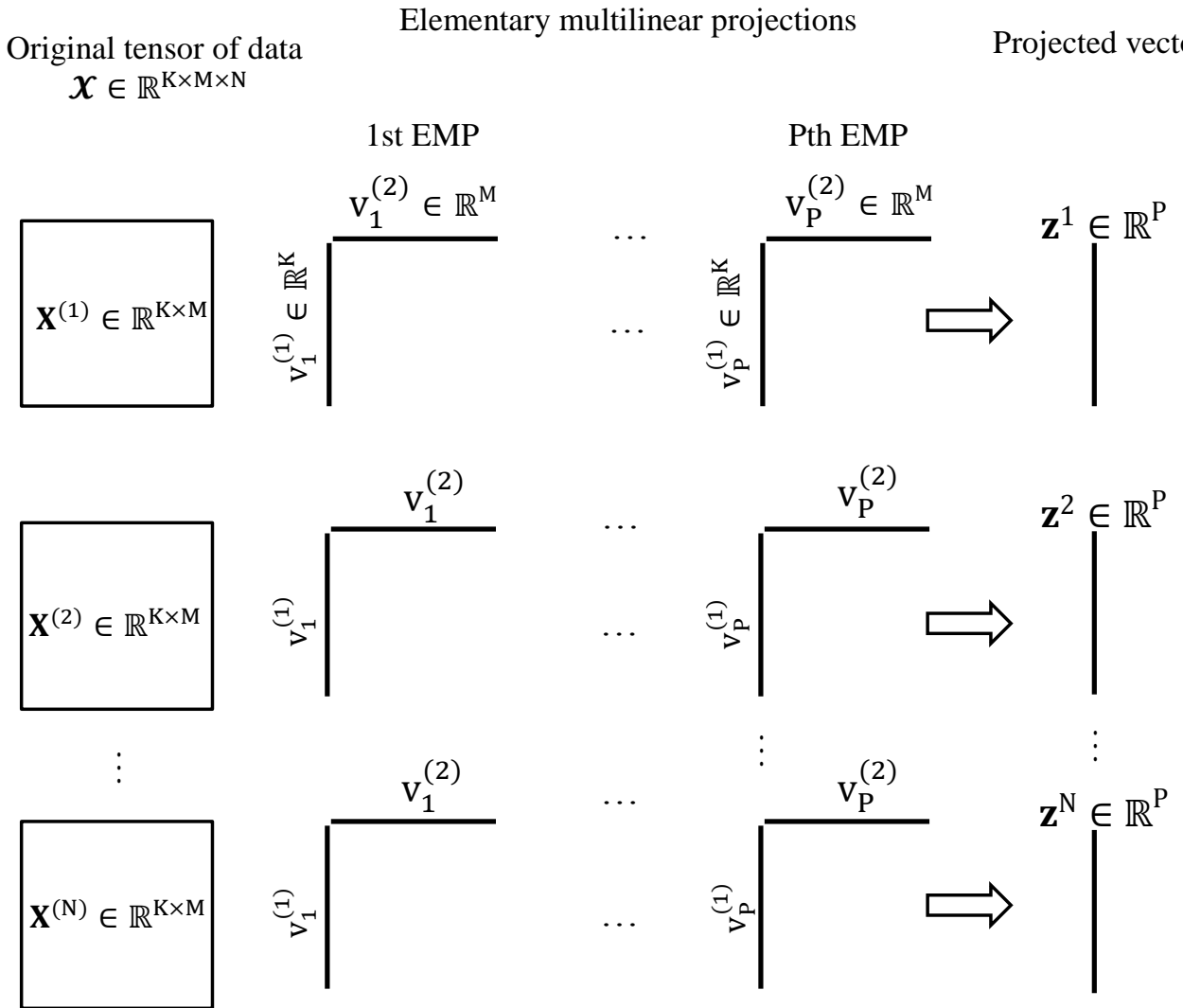

Figure 2: A visualization of the UMPCA method

Unfortunately, there is no closed form solution to this problem. One common approach to solve this problem is to assume that the projection vectors for all modes are pre-known except for one mode and solve the problem for that mode. Then, this procedure is repeated iteratively until convergence. A detailed algorithm to solve the UMPCA problem is provided by Lu et al. (2009). The relative importance of each EMP can be evaluated as the ratio of the explained variance by that EMP to the total explained variance i.e.

$$
\mathrm{R}_{\mathrm{p}}=\frac{\mathrm{s}^{\mathrm{p}}}{\sum_{\mathrm{p}=1}^{\mathrm{P}} \mathrm{s}^{\mathrm{p}}} \times 100 \%
$$


where $\mathrm{R}_{\mathrm{p}}$ is the relative importance metric for the $p^{\text {th }}$ EMP, and $\mathrm{s}^{\mathrm{p}}$ is the variance of the $p^{\text {th }}$ transformed vector. The $p^{\text {th }}$ eigentensor for the mode of the joint and trajectories can be defined as

$$
\mathrm{V}_{\mathrm{p}}^{(1,2)} \in \mathbb{R}^{\mathrm{K} \times \mathrm{M}}=\mathrm{v}_{\mathrm{p}}^{(1)} \circ \mathrm{v}_{\mathrm{p}}^{(2)}
$$

where $v_{p}^{(1)} \circ v_{p}^{(2)}$ is the outer product of the projection vectors of the joint and trajectory. These eigentensors combine all the information provided by the projection vectors in the selected modes.

\section{Results}

\subsection{Performance Comparison between PCA and UMPCA Using Simulation}

This section presents a simulation study that was conducted to show the advantage of using UMPCA over PCA for tensorial data. For this purpose, a set of surrogated tensorial data was generated based on real ingress motion profiles, which are represented by a third-order tensor $\mathcal{Y}$. Mode 1 represents the different joints; Mode 2, the joint trajectories; and Mode 3, different participants. The motion data of $\mathrm{K}=4$ joints for $\mathrm{N}=100$ participants are simulated. Each joint is represented by a trajectory with $M=600$ points, of which 200 points represent each of the directions $X, Y$, and $Z$. An element in tensor $\mathcal{Y}$ is denoted by $y_{\mathrm{ijk}}(\mathrm{i}=1, \ldots, 4 ; \mathrm{j}=1, \ldots, 600 ; \mathrm{k}=1, \ldots, 100)$, which corresponds to the $j^{\text {th }}$ point on the trajectory of joint $\mathrm{i}$ with regard to participant $\mathrm{k}$.

The purpose of this simulation is to illustrate how UMPCA is used to systematically find important joints capable of representing and inferring the major motion variation patterns. The simulation condition is set such that Joint 1 has the highest variability among all other joints, Joint 2 has the second largest variance, and Joints 3 and 4 have exactly the same variance, i.e., $\sigma_{\mathrm{j}_{1}}^{2}>\sigma_{\mathrm{j}_{2}}^{2}>\sigma_{\mathrm{j}_{3}}^{2}=\sigma_{\mathrm{j}_{4}}^{2}$, where $\sigma_{\mathrm{j}_{\mathrm{i}}}^{2}$ is 
the variance of Joint i. Furthermore, Joints 1 and 2 are positively correlated, whereas Joints 3 and 4 are negatively correlated. The joint trajectories are simulated by using a mixed-effect model that is defined as

$$
Y^{(k)}=X(B+R)+E
$$

where $\mathrm{Y}^{(\mathrm{k})}$ is the $\mathrm{M} \times \mathrm{K}$ matrix of the trajectories for the $k^{\text {th }}$ participant, i.e., the $i^{\text {th }}$ column of matrix $\mathrm{Y}^{(\mathrm{k})}$ gives the trajectories of the $i^{\text {th }}$ joint for participant $\mathrm{k}$. $\mathrm{X}$ is the $\mathrm{M} \times \mathrm{L}$ matrix of $\mathrm{B}$-spline basis values with $\mathrm{L}$ knots. A more realistic simulation dataset is obtained by using surrogate B-spline coefficients, which were obtained from real ingress trajectories, to generate the simulated trajectories, in which the trajectory of each joint is reconstructed using $\mathrm{L}=9$ knots. The matrix of fixed-effect coefficients is denoted by $B \in \mathbb{R}^{\mathrm{L} \times \mathrm{K}}$ and that of random effect coefficients, by $R \in \mathbb{R}^{\mathrm{L} \times \mathrm{K}}$. Each row in matrix $\mathrm{R}$ is normally distributed with a zero mean vector and a covariance matrix $\mathrm{U} \in$ $\mathbb{R}^{\mathrm{K} \times \mathrm{K}}$; the elements of matrix $\mathrm{U}$ can be obtained based on the variability of each joint and the covariance between different joints. Furthermore, matrix $E \in \mathbb{R}^{\mathrm{M} \times \mathrm{K}}$ is the vector of random errors following a normal distribution with a zero mean vector and a diagonal covariance matrix $\sigma_{\varepsilon}^{2} \mathrm{I}$ with $\sigma_{\varepsilon}^{2}=0.5$. The random-error matrix $\mathrm{E}$ is assumed to be independent of the random-effect matrix $\mathrm{R}$.

The generation of the simulation trajectories enables us to compare the UMPCA and PCA results to determine which method can more effectively capture the variation of motion patterns and the cross-correlation among different trajectories. Specifically, upon applying the UMPCA method to the simulated data, the relative importance $R_{p}$ of the first four components was $54.62 \%, 32.09 \%, 11.19 \%$, and $2.09 \%$, respectively. Because the first two components explain about $86 \%$ of the variability, this analysis focuses on these two components. 
Figure 3 shows the first and second eigentensors of the UMPCA method. Figure 3(a) indicates that the first eigentensor assigns higher weights to Joints 1 and 2 and almost zero weights to Joints 3 and 4 . The weights of the first joint are slightly larger than those of the second joint during the whole motion. This shows consistency with the simulation condition of $\sigma_{\mathrm{j}_{1}}^{2}=1000, \sigma_{\mathrm{j}_{2}}^{2}=900$, and $\sigma_{\mathrm{j}_{3}}^{2}=\sigma_{\mathrm{j}_{4}}^{2}=300$. Moreover, as the variability of Joints 3 and 4 is smaller than that of Joints 1 and 2, the first eigentensor only focuses on the prevalent Joints 1 and 2. Figure 3(b) shows the second eigentensor; it suggests a contrast between Joints 3 and 4, which is quite obvious when considering the motion in its entirety. This verifies the strong negative correlation $\rho_{j_{3} j_{4}}=-0.8$. Figure 4 shows the first and second eigenvectors of the joints mode obtained by UMPCA. As expected, in the first eigenvector, Joints 1 and 2 have higher weights compared to those of Joints 3 and 4. In the second eigenvector, it is clear that Joints 3 and 4 have the highest weights with opposite signs, highlighting the negative correlation between these joints.

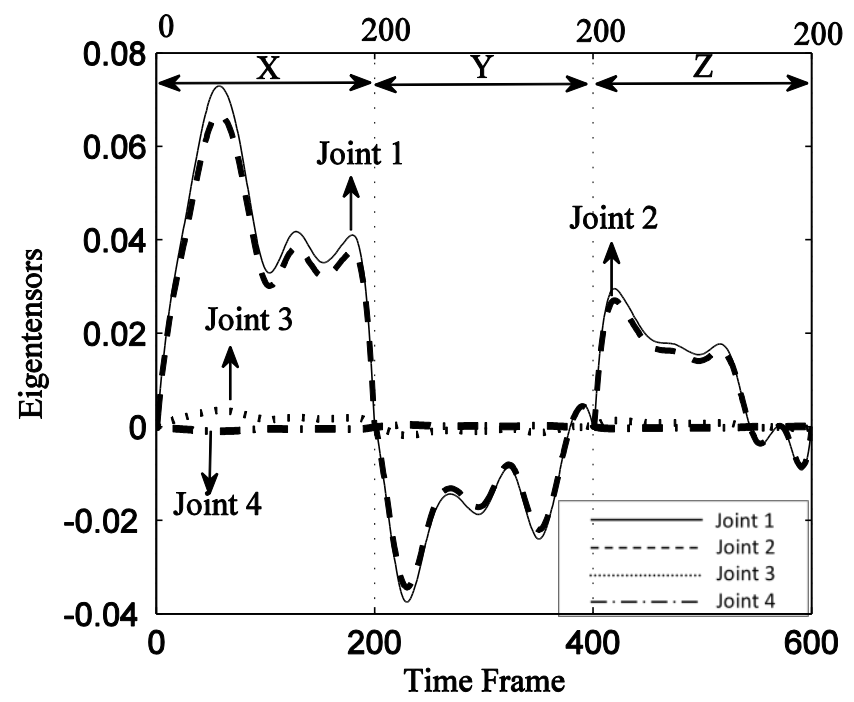

(a) First eigentensor 


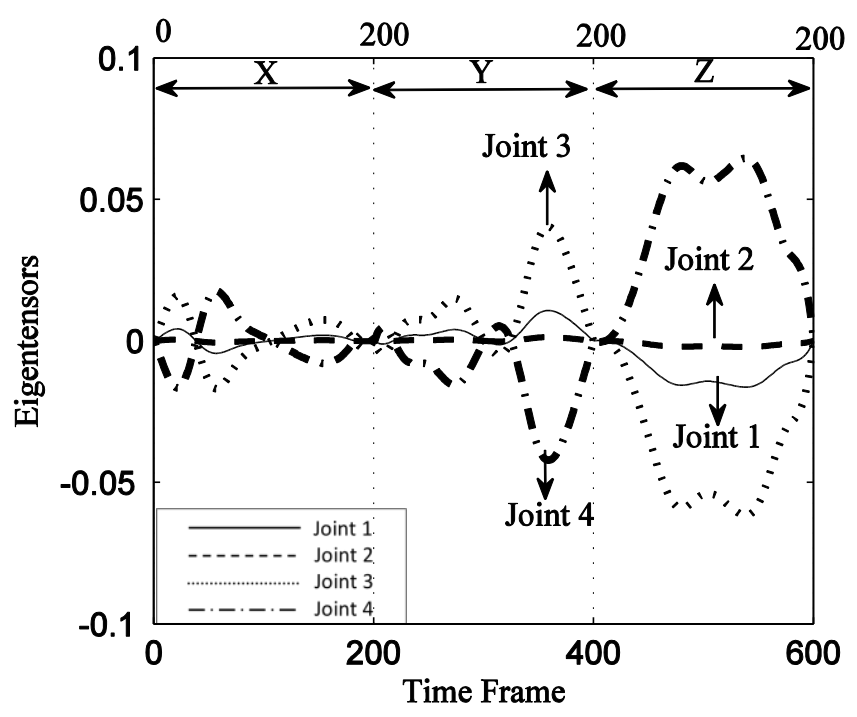

(b) Second eigentensor

Figure 3: UMPCA eigentensors

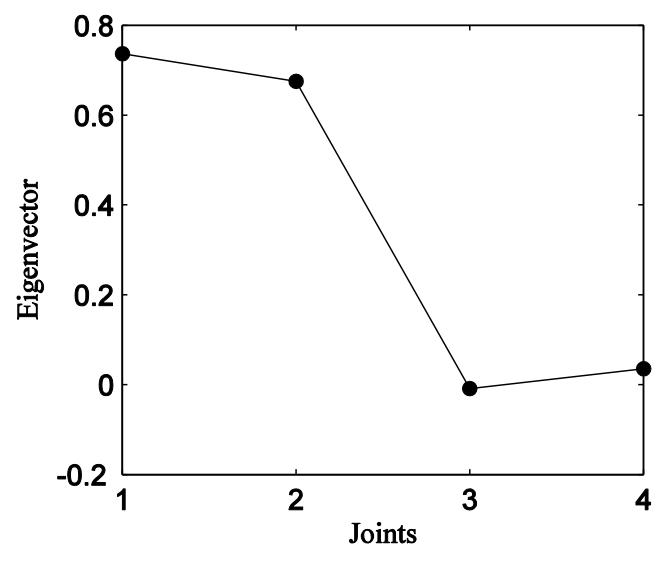

(a) First eigenvector

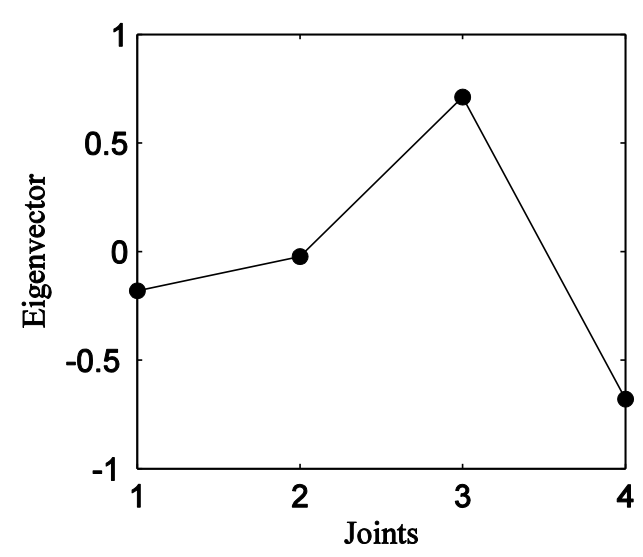

(b) Second eigenvector

Figure 4: UMPCA joint mode eigenvectors

For the comparison between the UMPCA and the PCA methods, we further apply the PCA method to the same simulated dataset. The resultant relative importance of the first four PC components is $29.04 \%, 28.66 \%, 22.36 \%$, and $19.95 \%$, respectively. Figure 5 shows the first and second eigenvectors as computed by the PCA method. It is noticeable in Figure 5(a) that there is no consistent significant joint that always show a higher weight than others. For example, although Joints 1 and 2 have higher weights in 
the $\mathrm{X}$-direction, Joint 3 has higher weights in some portions of the Y-direction.

Accordingly, we cannot conclude that only Joints 1 and 2 significantly contribute to the first eigenvector in all three directions.

Figure 5(b) shows the second eigenvector of the PCA method. This eigenvector, similar to the first eigenvector estimated by PCA, emphasizes the importance of Joints 1 and 2, unlike the UMPCA result that was able to clearly identify a new motion pattern that emphasizes the negative correlation between Joints 3 and 4. Furthermore, the tensorial representation utilized by the UMPCA method enables users to calculate the eigenvectors of the joint modes separately (see Figure 4), which provides the ability for a clearer and less subjective interpretation of the data. This simulation shows that the UMPCA method is more effective than PCA in analyzing multi-stream signals, especially in cases in which cross-correlations among signals exist.

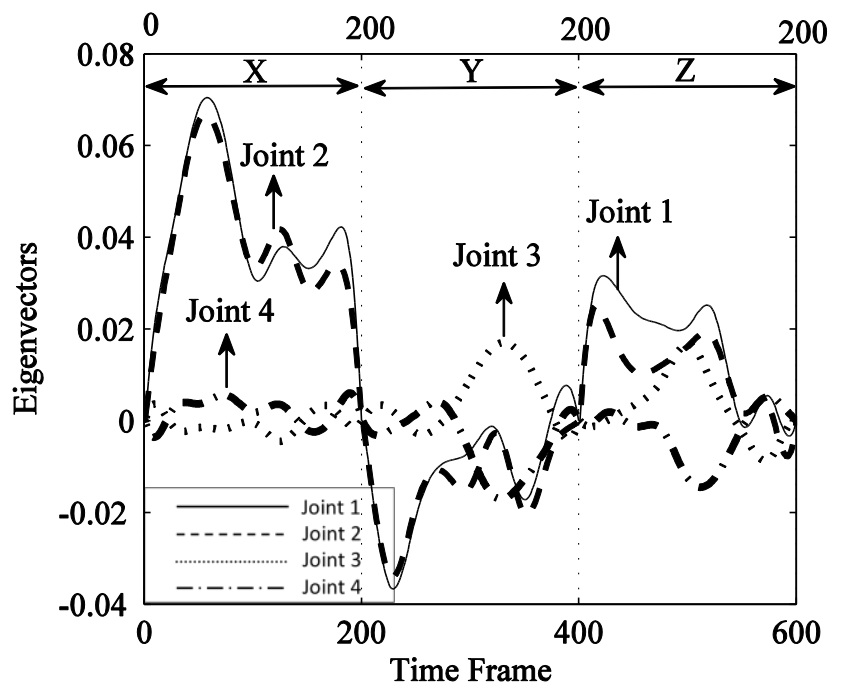

(a) First eigenvector 


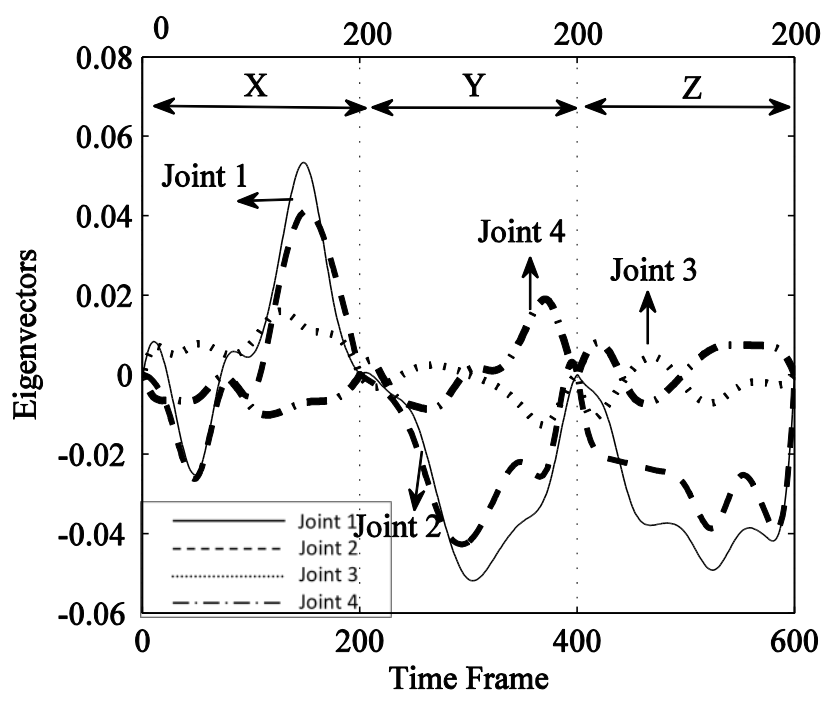

(b) Second eigenvector

Figure 5: First and second eigenvectors computed by PCA method

\subsection{Case Study: Identifying Important Ingress Motion Patterns}

In this subsection, the UMPCA method is applied to analyze experimental data relating to ingress motion.

\subsubsection{Data Source}

An automotive manufacturer performed experimental tests on different types of vehicles with the purpose of capturing the motion of participants during ingress and egress. The 32 participants chosen in the study are representative of the general population spanning from a $3 \%$ female to a $98 \%$ male in height (stature). The participants varied in body mass index from very thin $(B M I=19)$ to obese $(B M I=52)$.

The ingress and egress motions were captured for 17 vehicle designs by adjusting 7 key design variables. These 7 design variables are defined as per the SAE J1100 standard and are shown in Figure 6. The programmable vehicle buck, the Human Occupant Package Simulator (HOPS), was used to create the vehicle designs. 15camera VICON motion capture system with a sampling frequency of $60 \mathrm{~Hz}$ were used and were strategically located to avoid obstructions between the cameras and body 
markers. The motion capture system was calibrated using the VICON iQ software (version 1.0.7, NY, USA).

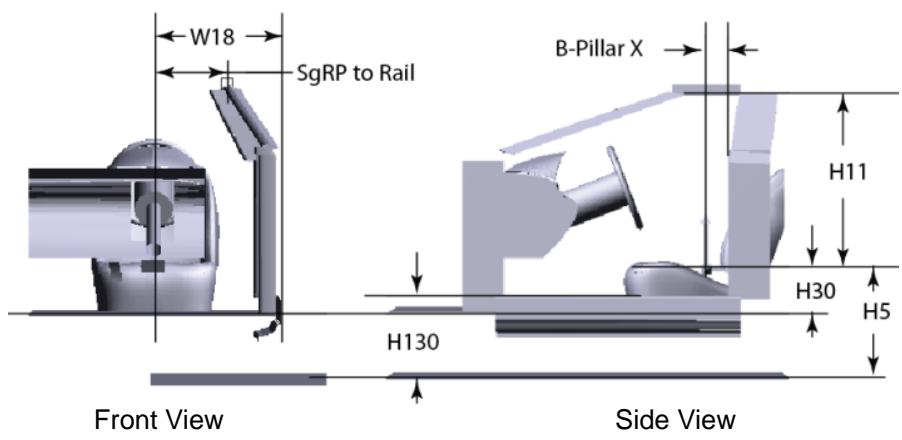

Figure 6: Car seat design variables as per SAE J1100

To capture the whole-body motion, a unique set of reflective markers were used. The markers were attached strategically to ensure that motion of the joints could be accurately reconstructed. The locations of 20 joints were then estimated, which are left hip, left ankle, left knee, left toe, left shoulder, left elbow, left wrist, left clavicle, right ankle, right knee, right toe, right shoulder, right elbow, right wrist, right clavicle, head, neck, and the spinal vertebrae T12L1, T1T2, S1L5. Figure 1(a) shows an example of the motion trajectories calculated from one ingress trial. The trajectories were expressed in a coordinate system with the $\mathrm{z}$ axis indicating vertical direction, $\mathrm{x}$ axis representing the oriented fore and aft along the vehicle longitudinal direction, and y axis showing the cross car direction.

Participants were asked to enter and exit the vehicle mockup as they would a vehicle. The right-leg-first strategy was used to define the start/end points for ingress trial (Chateauroux 2009, Masoud et al. 2016). After completing an ingress/egress trial, participants were asked to rate the ease of getting in and out the vehicle. The responses on a 10-point scale were 1-2 (unacceptable), 3-5 (average), 6-8 (outstanding), and 9-10 (truly exceptional). 


\subsubsection{Case study results}

In this case study, the human motion data of 15 subjects performing ingress in two different vehicle designs were chosen for analysis. In the original experiment, each participant did not test both vehicle designs, and there are only 15 subjects that tested both vehicle designs. Of those 15 subjects, 7 were female and 8 were male.

Furthermore, the subjects' BMI ranged from a 34.5 to 21 and stature ranged from $3 \%$ female to a $98 \%$ male. The two vehicle designs were chosen such that one was comfortable to ingress (i.e. average ingress rating of subjects $=7.7, \min =5, \max =9$ ) and the other was difficult to ingress (i.e. average ingress rating of subjects $=2.5$, $\min =1, \max =6$ ). Table 1 lists the most important anthropometric features of the participants with their associated distributional parameters. The trajectory data are represented by the third-order tensor $X^{20 \times 600 \times 30}$, where the three tensor modes correspond to the joints, trajectories, and participants, respectively.

Table 1: Distributional parameters of the participants' anthropometric variables

\begin{tabular}{cccc}
\hline Anthropometric variable & Mean & $\begin{array}{c}\text { Standard } \\
\text { deviation }\end{array}$ & Range \\
\hline Stature (mm) & 1705.6 & 123.4 & $1492-1924$ \\
\hline Sitting height (mm) & 901.9 & 60.9 & $806-1022$ \\
\hline Sitting height / Stature & 0.53 & 0.015 & $0.48-0.56$ \\
\hline Weight & 81.1 & 23.4 & $52-136$ \\
\hline BMI & 27.7 & 7.4 & $19-52$ \\
\hline Age & 42.9 & 9.3 & $24-58$
\end{tabular}

The UMPCA method was applied to this tensor data as a systematic way to automatically identify those motion patterns with high variation in the ingress motion and also to identify which motion pattern is associated with ingress comfort. Table 2 lists the relative importance of the first ten UMPCA components with the important 
joints selected using a clustering method which will be discussed later on. It can be observed that the first UMPCA component is the most important component with a relative importance of $79.44 \%$. Hence, our subsequent inference analysis only focuses on this first component.

Table 2: Relative importance and selected important joints of UMPCA components applied to human motion data

\begin{tabular}{c|c|l}
\hline \multicolumn{1}{c}{ UMPCA Components } & Relative Importance & \multicolumn{1}{c}{ Selected joints } \\
\hline 1 & $79.44 \%$ & $\begin{array}{l}\text { head, neck, T1T2, right shoulder, left } \\
\text { shoulder, right clavicle, left clavicle, } \\
\text { and left elbow }\end{array}$ \\
\hline 2 & $12.75 \%$ & $\begin{array}{l}\text { Right Ankle, Left Ankle, Left Knee, } \\
\text { Left Elbow }\end{array}$ \\
\hline 3 & $4.31 \%$ & $\begin{array}{l}\text { Right Hip, Left Hip, Pelvis, T12L1, } \\
\text { S1L5, Right Elbow }\end{array}$ \\
\hline 4 & $1.30 \%$ & $\begin{array}{l}\text { Right Hip, Left Ankle, Right Knee, } \\
\text { Left Toe }\end{array}$ \\
\hline 5 & $0.90 \%$ & Right Elbow \\
\hline 7 & $0.47 \%$ & $\begin{array}{l}\text { All joints except Right Ankle, Right } \\
\text { Knee, Right Toe, Right Elbow, Left } \\
\text { Knee }\end{array}$ \\
\hline 5 & $0.31 \%$ & Left Elbow \\
\hline \multirow{2}{*}{10} & $0.25 \%$ & $\begin{array}{l}\text { Right Hip, Right Ankle, Left Ankle, } \\
\text { T12L1, Right Knee, Right Shoulder, } \\
\text { Right Elbow, Left Knee, Left Toe, } \\
\text { Left Shoulder, Left Elbow }\end{array}$ \\
\hline & $0.12 \%$ & $\begin{array}{l}\text { Right Hip, Right Ankle, Left Ankle, } \\
\text { T12L1, Right Knee, Right Shoulder, } \\
\text { Right Elbow, Left Knee, Left Toe, } \\
\text { Left Shoulder, Left Elbow }\end{array}$ \\
\hline & $0.05 \%$ & $\begin{array}{l}\text { Right Ankle, Head, Right Toe, Right } \\
\text { Shoulder, Left Knee, Right Clavical }\end{array}$ \\
\hline
\end{tabular}

Figure 7 shows a plot of the eigenvector weights of the first component obtained using UMPCA. The joint mode eigenvector shown in Figure 7(a) shows the joint contributions among 20 joints, and the trajectory mode eigenvector shown in Figure 7(b) shows the importance among time frames 1-200 in each of the X-, Y-, and Zdirections. The important joints and time frames that significantly contribute to the UMPCA component are systematically identified by applying the hierarchical clustering method (Hastie et al. 2009) to each eigenvector. This clustering method determines the decision boundaries to automatically remove unimportant small weights in each 
eigenvector, which fall within the shadow region in Figure 7. Specifically, Figure 7(a) shows that only 8 joints (i.e., head, neck, T1T2, right shoulder, left shoulder, right clavicle, left clavicle, and left elbow) are important joints beyond the shadow region. Figure 7(b) shows that only the time frames $97-132$ in the X-direction and 42-200 in the Z-direction are important. This is explicitly interpreted to indicate that the first UMPCA component mainly represents the motion of the upper body in the longitudinal and vertical moving directions as the participant lower their head to enter the vehicle. Moreover, Figure 7(b) can further facilitate the identification of the specific time frames in which this motion pattern is most evident (i.e., the most significant variation pattern in the $\mathrm{X}$ - and Z-direction occurs at time frame $\mathrm{t}_{\mathrm{x}}^{*}=111$ and $\mathrm{t}_{\mathrm{z}}^{*}=79$, respectively).

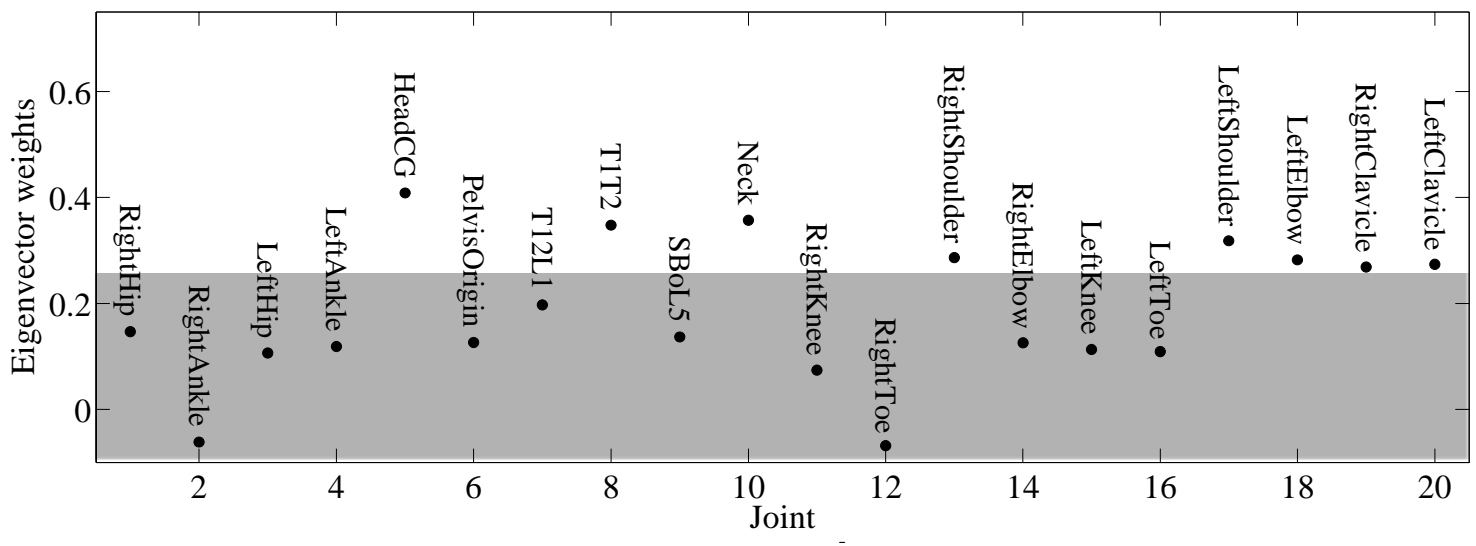

(a) First eigenvector - joint mode

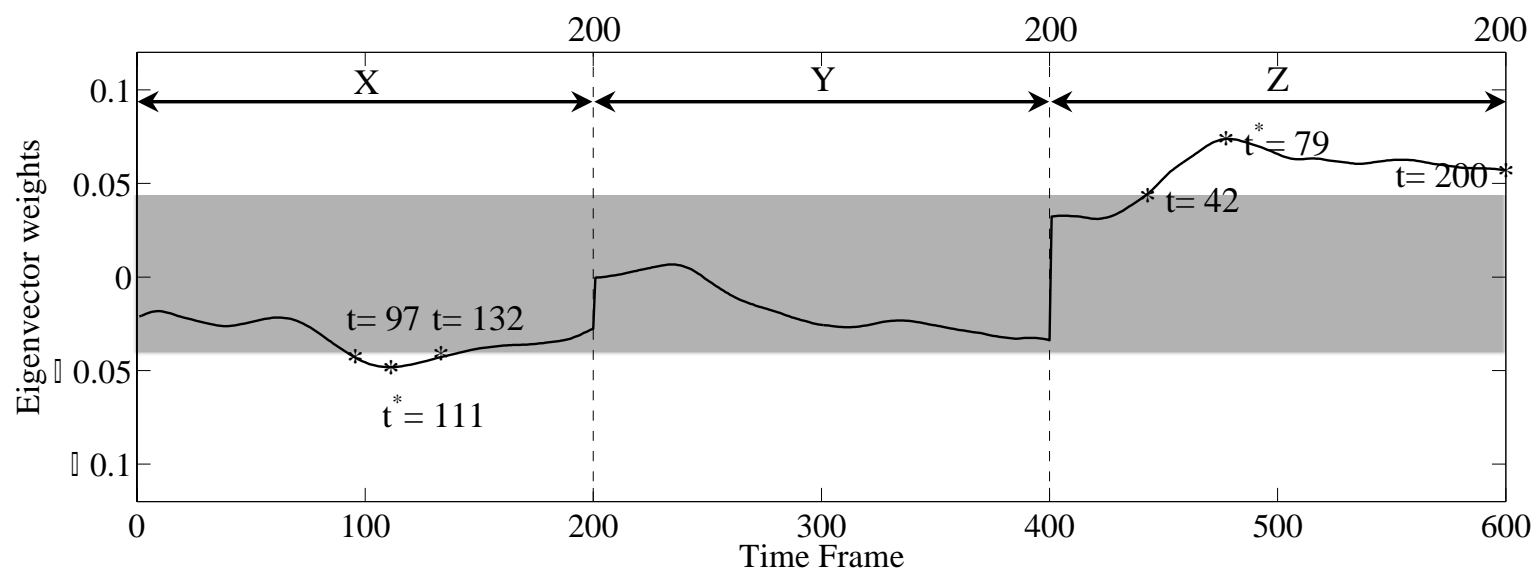

(b) First eigenvector - trajectory mode

Figure 7: Eigenvectors of the first UMPCA component 
We verified these facts by plotting several time frames of one participant performing the ingress motion for both vehicles in Figure 8 and Figure 9, respectively, where the selected time frames correspond to the points identified in Figure 7. We can observe that Figure 8 shows inference results consistent with those of Figure 7, that is, the most significant variation between the upper body joints in the X-direction occurs at time frames $97-132$. In this case, the variation at $t_{x}^{*}=111$ is similar to that at time frames 97 and 132 due to their similar eigenvector weights. Similarly, Figure 9 shows that the most significant variation in the Z-direction occurs during time frames 42-200 and is most evident at $t_{z}^{*}=79$. This shows the advantage of using the UMPCA method, which obviates the need for tedious visual efforts in finding the specific time frames of the most significant motion patterns.

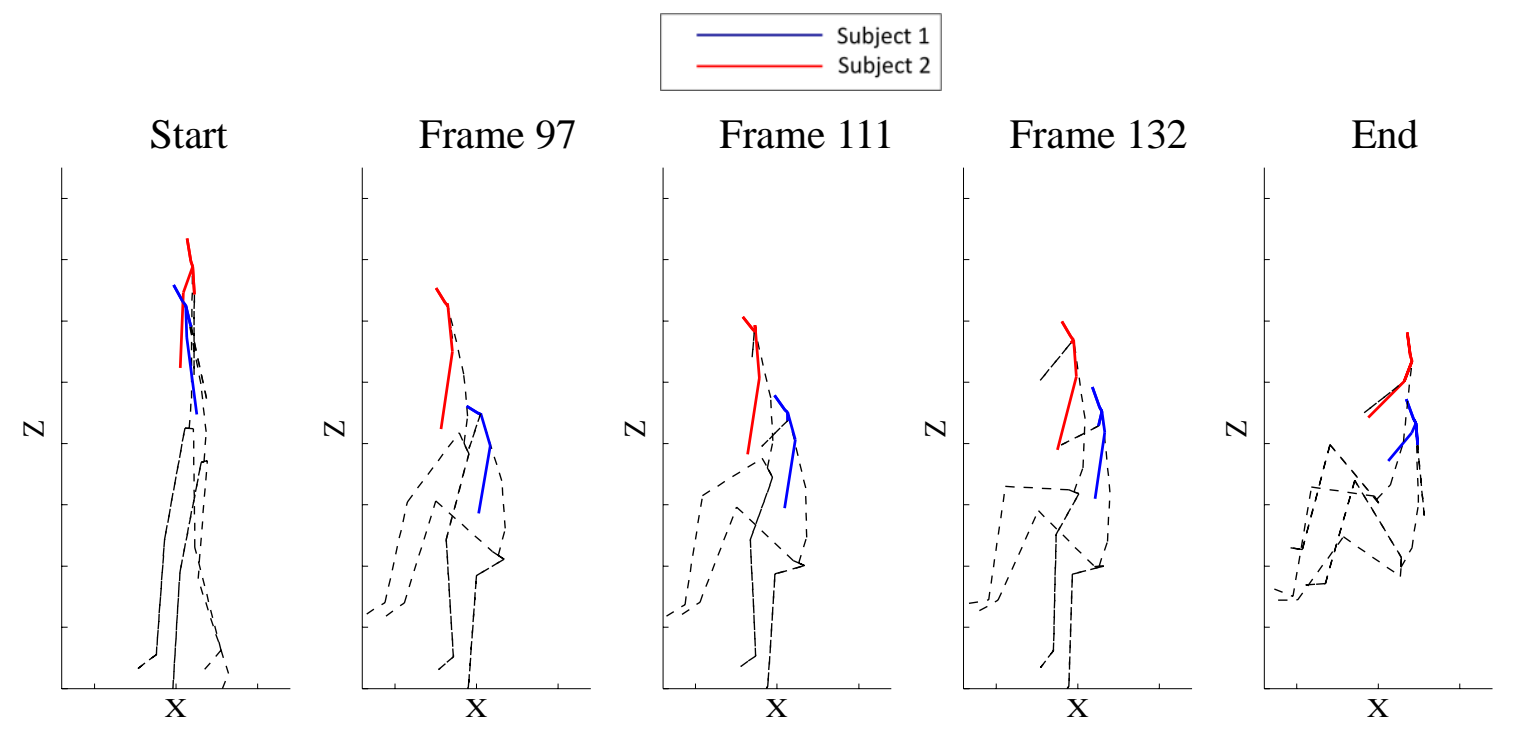

Figure 8: Ingress motion - joints and time frames identified by the first UMPCA component in the $\mathrm{X}$-direction 


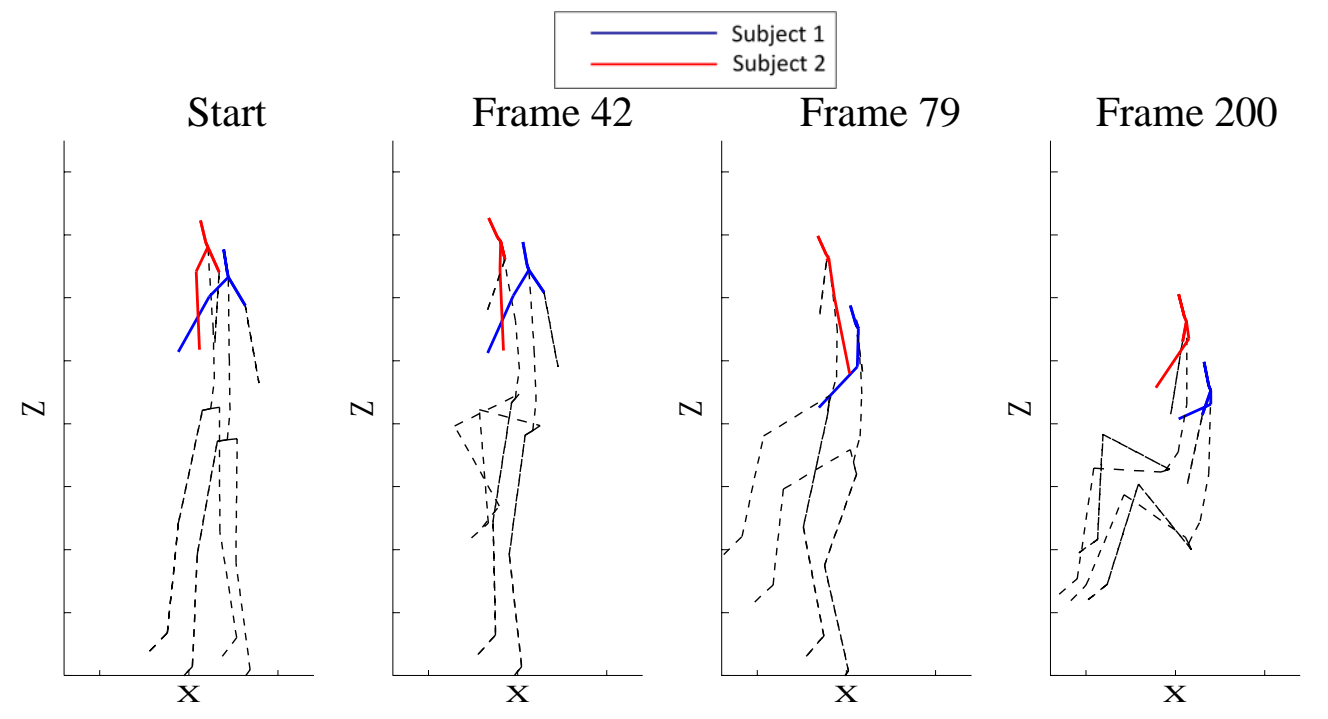

Figure 9: Ingress motion - joints and time frames identified by the first UMPCA component in the Z-direction

The effect of the first motion pattern on discomfort was studied by plotting the UMPCA scores of the first and second UMPCA components (Figure 10). The scores associated with the first vehicle design, which has a comfortable ingress, are denoted by crosses, and the scores associated with the second vehicle design, which has a less comfortable ingress, is denoted by circles. It can be observed that the first UMPCA component clearly separates the two vehicle designs, thus showing that the first motion pattern has a strong effect on the ingress comfort. To further confirm that the two car designs are significantly separated, a t-test on the mean parameters is performed on the UMPC score and the P-value is computed as $1.73 \mathrm{E}-12$. As a result, the difference in the UMPC scores for first and second car configurations is statistically significant. This information enables vehicle designers to study the vehicle design parameters that have an effect on this motion pattern, and implement design changes to improve ingress comfort. 


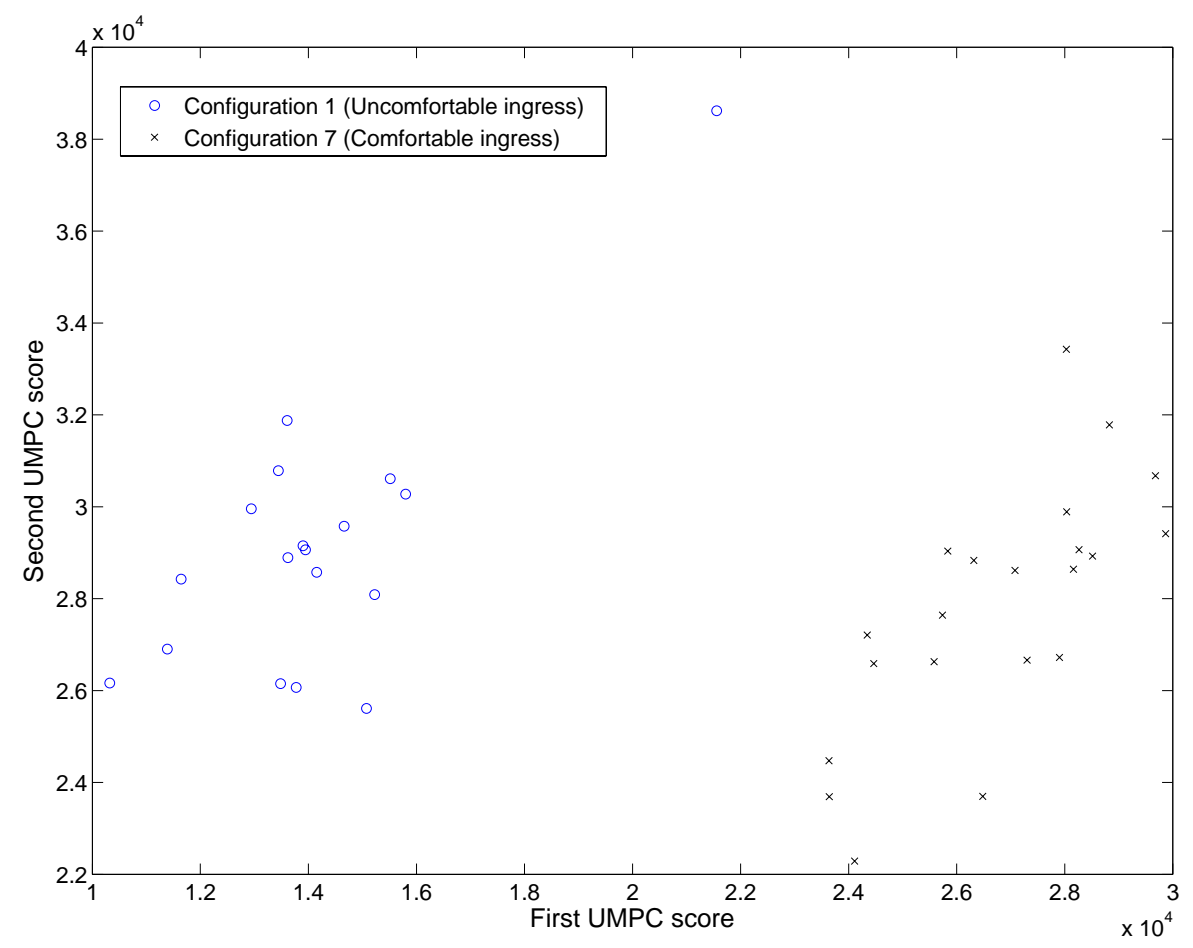

Figure 10: First and second UMPCA scores for two vehicle configurations

To further demonstrate the performance of the UMPCA method used in this paper, we applied the PCA method on the same dataset. In order to apply PCA on the dataset, the third-order tensor $\mathcal{X}^{20 \times 600 \times 30}$ was transformed into a matrix $\mathrm{X}^{30 \times 12000}$ where the rows represent the number of samples and the columns represent the trajectory data of 20 joints (i.e. 600 points per trajectory x 20 joints). Figure 11 shows a plot of the eigenvector weights of the first component obtained using PCA, where each 600 points in the $\mathrm{x}$ axis represent the trajectory data of one joint. To identify the time frames that significantly contribute to the PCA component the same hierarchical clustering method used in the UMPCA method was applied. Figure 11 shows that 18 out of the 20 joints are identified as important (i.e. beyond the shadow region). This result shows that the PCA method fails to identify a clear pattern in the first component. 


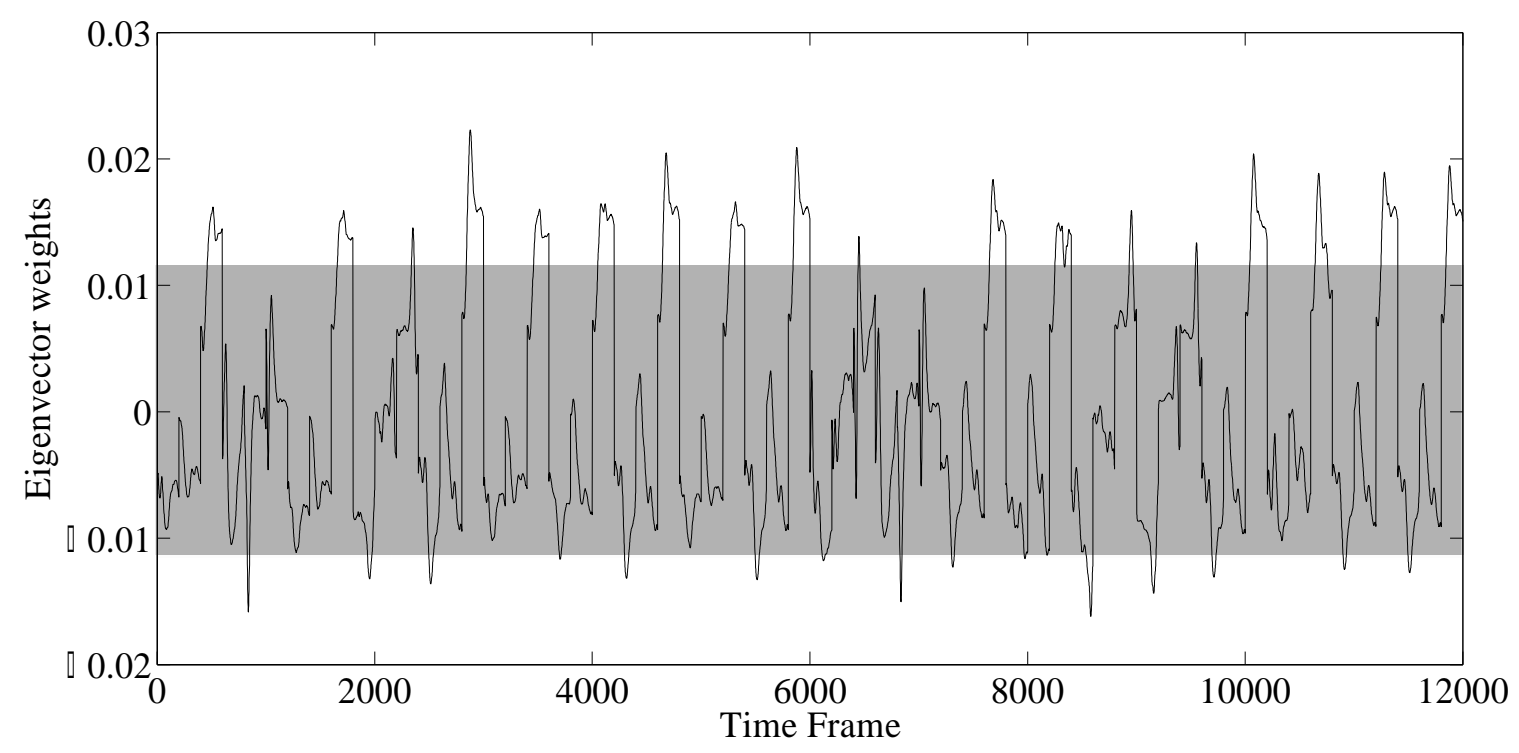

Figure 11: Eigenvector of the first PCA component

\section{Conclusion}

This paper proposes a method for identifying important human motion variation patterns using UMPCA. Unlike PCA, this method preserves the multistream structure of the motion data. A simulation study was conducted to demonstrate the superiority of UMPCA over PCA. The simulation results showed that UMPCA can capture the crosscorrelation among different signals and important variation patterns more effectively than PCA. UMPCA was also applied to a case study for vehicle ingress motion analysis, where the motion pattern that has the highest variation was identified. We further demonstrate that this motion pattern is associated with ingress discomfort, although this may have been a coincidence due to the particular test conditions selected. The information about this motion pattern can be used to guide and improve vehicle design.

This work involved the acquisition of human motion data using marker-based technologies, as explained in the introduction. The proposed method, however, is not restricted to marker-based trajectory data; rather, it can also be applied to other types of multistream trajectories. For example, markerless motion capture systems (Corazza et al. 2006) have provided an opportunity to capture human motion trajectories without 
using markers. Moreover, advances in computer vision technologies (Wang et al. 2003) have also enabled the capture of human motion trajectories using cameras that are not necessarily located in an experimental setting, such as surveillance cameras.

The proposed method can also be applied to various other human motion datasets such as those representing athletic performance evaluation and training, medical diagnosis, and video surveillance. This method can also be integrated into Gait analysis software packages to produce more consistent and reliable results for clinical diagnosis. In the future, we plan to develop a UMPCA-based method capable of producing sparse eigenvectors. This could further enhance the interpretability of the eigenvectors.

\section{Acknowledgements}

This work was partially supported by the National Science Foundation (Grant No.

CMMI-1233108). The authors would like to thank Ford for providing the test data and Dr. Matt Reed for his help on the work. In addition, the constructive comments and suggestions from reviewers and the editor are greatly appreciated.

\section{References}

Bharati, M. H., J. J. Liu and J. F. MacGregor (2004). "Image Texture Analysis:

Methods and Comparisons." Chemometrics and intelligent laboratory systems 72(1), pp. 57-71.

Chateauroux, E. (2009). "Analyse du Mouvement d'accessibilité au Poste de Conduite d'une Automobile en vue de la Simulation-Cas Particulier des Personnes Âgées." Ph.D. thesis, INSA Lyon, France.

Cook, R. E., I. Schneider, M. E. Hazlewood, S. J. Hillman and J. E. Robb (2003). "Gait Analysis Alters Decision-Making in Cerebral Palsy." Journal of pediatric orthopaedics 23(3), pp. 292-295.

Corazza, S., L. Muendermann, A. Chaudhari, T. Demattio, C. Cobelli and T. P. Andriacchi (2006). "A Markerless Motion Capture System to Study Musculoskeletal Biomechanics: Visual Hull and Simulated Annealing Approach." Annals of biomedical engineering 34(6), pp. 1019-1029. 
Hastie, T., R. Tibshirani, J. Friedman, T. Hastie, J. Friedman and R. Tibshirani (2009). The Elements of Statistical Learning. Springer, New York, pp. 472-479.

He, X., D. Cai and P. Niyogi (2005). "Tensor Subspace Analysis." Advances in neural information processing systems.

Kay, R. M., S. Dennis, S. Rethlefsen, D. L. Skaggs and V. T. Tolo (2000). "Impact of Postoperative Gait Analysis on Orthopaedic Care." Clinical orthopaedics and related research 374, pp. 259-264.

Knudson, D. V. (2013). Qualitative Diagnosis of Human Movement: Improving Performance in Sport and Exercise. Human Kinetics, pp. 16-38.

Kolda, T. G. and B. W. Bader (2009). "Tensor Decompositions and Applications." SIAM review 51(3), pp. 455-500.

Li, H., S. Wu, S. Ba, S. Lin and Y. Zhang (2006). Advances in Multimedia Modeling. Springer, New York, pp. 73-82.

Lu, H., K. N. Plataniotis and A. N. Venetsanopoulos (2009). "Uncorrelated Multilinear Discriminant Analysis with Regularization and Aggregation for Tensor Object Recognition." Neural Networks, IEEE Transactions on 20(1), pp. 103-123.

Lu, H., K. N. Plataniotis and A. N. Venetsanopoulos (2009). "Uncorrelated Multilinear Principal Component Analysis for Unsupervised Multilinear Subspace Learning." Neural Networks, IEEE Transactions on 20(11), pp. 1820-1836.

Mason, C. R., J. E. Gomez and T. J. Ebner (2001). "Hand Synergies During Reach-toGrasp." Journal of Neurophysiology 86(6), pp. 2896-2910.

Masoud, H. I., M. P. Reed, K. Paynabar, J. J. Jin, K. K. Kozak, N. Wang, J. Wan and G. Gomez-Levi (2016). "Predicting Subjective Responses from Human Motion:

Application to Vehicle Ingress Assessment." Journal of Manufacturing Science and Engineering 138(6): 061001.

Paynabar, K., J. Jin and M. Pacella (2013). "Monitoring and Diagnosis of Multichannel Nonlinear Profile Variations Using Uncorrelated Multilinear Principal Component Analysis." IIE Transactions 45(11), pp. 1235-1247.

Wang, L., W. Hu and T. Tan (2003). "Recent Developments in Human Motion Analysis." Pattern recognition 36(3), pp. 585-601.

Yan, H., K. Paynabar and J. Shi (2015). "Image-Based Process Monitoring Using LowRank Tensor Decomposition." 